\title{
Main-phase creation of "seed" electrons in the outer radiation belt
}

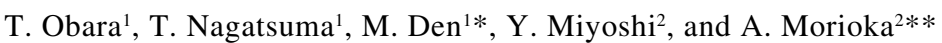 \\ ${ }^{1}$ Hiraiso Solar Terrestrial Research Center, CRL, Hitachinaka, Ibaraki 311-1202, Japan \\ ${ }^{2}$ Planetary Plasma and Atmospheric Research Center, Tohoku University, Sendai 980-8578, Japan \\ (Received May 11, 1999; Revised November 25, 1999; Accepted November 25, 1999)
}

\begin{abstract}
During a geomagnetic storm in early November 1993, NOAA satellite observations revealed that a population of energetic electrons appeared in the center of the outer radiation belt during the main phase of the storm. At the beginning of the main phase of the magnetic storm, the number of electrons with energies from $30 \mathrm{keV}$ to $100 \mathrm{keV}$ increased rapidly and contributed to build up of the ring current. At the end of the main phase the flux of electrons with energies greater than $300 \mathrm{keV}$ increased significantly. Akebono satellite observations showed that the flux of electrons with energies ranging from $300 \mathrm{keV}$ to $950 \mathrm{keV}$ increased late of the storm main phase and that the flux of electrons with energies from $950 \mathrm{keV}$ to $2.5 \mathrm{MeV}$ increased during the storm recovery phase. The electron flux increase observed by both NOAA and Akebono took place first in the central part of the outer radiation belt $(L \sim 4)$ and propagated to higher $L$ shells with a significant time delay. We think that the ring current electrons that appeared first and near $L \sim 4$ during the main phase seeded the subsequent increase in the flux of $\mathrm{MeV}$ electrons in the entire outer radiation belt.
\end{abstract}

\section{Introduction}

Very drastic changes in the outer radiation belt electrons during magnetic storms were observed by McIlwain (1966), who reported that the electron flux in the outer radiation belt at altitude of geosynchronous orbit decreases at the beginning of the main phase of the magnetic storm. Analyzing the SAMPEX and GPS observations, Li et al. (1997) found that during the main phase of a magnetic storm the relativistic electron flux decreases not only at the geosynchronous altitude but also near the peak portion of the outer radiation belt $(L \sim 4)$. It was reported by Obara et al. (1998) that energetic electron enhancements above pre-storm levels occur in the outer radiation belt during the geomagnetic storm.

Although the radiation belt has been studied since its discovery in 1958, many issues remain unclear not only because of the early lack of high-resolution data but also because the subject was of little interest. The importance of relativistic electrons to the Space Weather, however, has recently been recognized, because large increases in the number of relativistic electrons in the outer radiation belt can disrupt the satellite operation (Baker et al., 1998).

The present paper, follow-up of the special paper "An overview of early November 1993 geomagnetic storm" by Knipp et al. (1998), intends to clarify the large enhancement of the relativistic electrons in the outer radiation belt during that storm by paying a particular attention to

\footnotetext{
*Present address: 3601, Isozaki, Hitachinaka, Ibaraki 311-1202, Japan.

**Present address: Aoba, Aramaki, Aoba-ku, Sendai 980-8578, Japan
}

Copy right $\odot$ The Society of Geomagnetism and Earth, Planetary and Space Sciences (SGEPSS); The Seismological Society of Japan; The Volcanological Society of Japan; The Geodetic Society of Japan; The Japanese Society for Planetary Sciences. "seed" population. Akebono and NOAA data have been used to demonstrate the main-phase creation of a population of energetic electrons near the center of outer radiation belt $(L \sim 4)$ that "seeded" the subsequent enhancement of the outer belt electrons. Possible processes that might make electrons more energetic are discussed.

\section{Background}

The early November 1993 event (Knipp et al., 1998) is one of particularly recurrent magnetic storms in the declining phase of solar cycle 22. It was characterized by a high-speed solar wind with IMF sector polarity change. An extension of the north polar coronal hole was identified as being responsible for the dominant high-speed stream and the IMF away orientation.

The solar wind velocity increased about 2000 UT on November 3 and reached $650 \mathrm{~km} / \mathrm{s}$ by about 0500 UT on November 4. Increases in both solar wind density and magnetic field intensity were associated with the beginning of the increased solar wind speed at 2200 UT on November 3 . The density reached more than one hundred/cc and the magnetic field intensity increased to $40 \mathrm{nT}$ (Knipp et al., 1998). A large southward excursion of the IMF with intensity of $\sim 35 \mathrm{nT}$ at $2300 \mathrm{UT}$ caused a major magnetic storm.

A rather steep decline of the Dst began as soon as the storm onset was recorded at 2200 UT. The Dst value reached a local minimum just before 0100 UT on November 4 , and the most negative value $(-116 \mathrm{nT})$ was recorded about 10 hours later, when the combination of the prolonged strong southward IMF and the very fast solar wind flow with high density produced strong ring current effects (Kozyra et al., 1998). The excursion to major storm was brief ( $\sim 5$ hours) and occurred with the second peak of main phase, consistent with the recent finding of Yokoyama 
and Kamide (1997). The recovery phase commenced at 1200 UT on November 4 (Knipp et al., 1998).

\section{Previous Works on Relativistic Electrons for this Storm}

Obara et al. (1998) investigated a behavior of the relativistic electrons with energies greater than $2 \mathrm{MeV}$ at geosynchronous location for this storm by evaluating the GMS/SEM observations. Figure 1 shows the variation of $\mathrm{MeV}$ electrons at GMS location. On almost each we can see flux maximum around 0200 UT, which can be understood a diurnal variation. Flux value was slightly low for most of November 3. After the sudden commencement particle flux peaked for the day. Between 2325 UT on November 3 and 0030 UT on November 4, the flux value dropped precipitously. The storm reached its maximum with a Dst value of $-116 \mathrm{nT}$ about $1200 \mathrm{UT}$ on November 4 and then started to weaken. The GMS satellite over Japan actually observed the return flux of the $2 \mathrm{MeV}$ population about 18 UT on November 4 . Over the subsequent days the flux level returned to the normal level and then exceeded it by more than an order of magnitude (Obara et al., 1998).

Li et al. (1997) made a "multi" satellite survey of the energetic particle environment for this storm by evaluating SAMPEX (low altitude), GPS (mid altitude) and LANL (geostationary) observations. The electron energy thresholds for SMPEX satellite are $E>1 \mathrm{MeV}$ and $E>3$ $\mathrm{MeV}$. GPS data showed the relativistic electrons with energies greater than $3 \mathrm{MeV}$ were depleted at $4<L<8$ for more than 12 hours after the onset of the storm. Li et al. (1997) further found the return flux appeared in low $L$ shells first and the less energetic particles appeared first. The returning flux exceeded pre-storm level by one order of magnitude at geosynchronous location, being consistent with Obara et al. (1998).

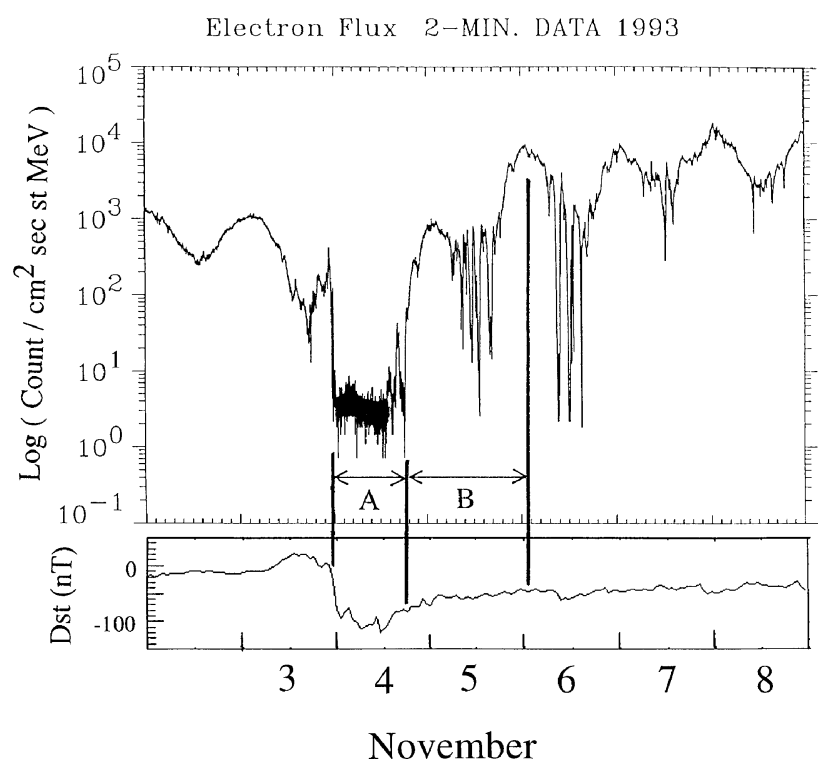

Fig. 1. Time variation of the MeV electron flux (top) and of the magnetic disturbance (Dst) index from November 2 to 8 (after Obara et al., 1998).
Nakamura et al. (1998) made a follow-up study based on SAMPEX electron observations. They found severe flux reduction at $4<L<7$ during the main phase of the storm and found a large enhancement during the recovery phase. They have tested an adiabatic deceleration/ acceleration processes by using a time varying magnetic field intensity, and concluded that some unknown processes are taking place in transporting electrons inward and getting electrons more energetic to interpret large enhancement of relativistic electrons in the outer radiation belt.

The following issues are still unclear:

(1) Where do the relativistic electrons go at the storm onset?

(2) What is the source population filling the entire outer belt during the storm recovery phase?

(3) What processes are responsible for making electrons more energetic ?

In this paper we concentrate on evaluating the Akebono and NOAA observations relevant to items (2) and (3).

\section{Akebono Observations}

The Akebono satellite was launched on February 22, 1989 into an initial orbit with inclination of $75 \mathrm{deg}$., an apogee of $10500 \mathrm{~km}$ and a perigee of $275 \mathrm{~km}$. A radiation monitor (RDM) instrument on the satellite monitored the relativistic particles (electrons, ions and alphas) in the Earth's magnetosphere. The RDM instrument consists of four solid state detectors that cover three energy ranges for electrons; i.e., 0.3-0.95 MeV, 0.95-2.5 MeV and more than $2.5 \mathrm{MeV}$. We used RDM data with a time resolution of $30 \mathrm{sec}$ in the work in this paper.

During early November, 1993, the Akebono satellite traversed the polar region from the dawnside sector to the duskside sector over the Antarctica Syowa base, to which real time telemetry data were sent. Figure 2 shows results of successive Akebono observations in the evening sector (MLT 17 h). Electron fluxes are plotted against $L$ value. On November 3 (see Fig. 2(a)) we can clearly identify the outer belt, the slot region and the part of the inner belt population. The peak portion of the outer belt located at $L=4$ and the slot region was seen around $L=2.7$ for November 3.

As seen in Fig. 2(c) the flux of outer belt electrons dropped drastically at the beginning of the magnetic storm. The highest-energy electrons $(>2.5 \mathrm{MeV})$ can hardly be seen in the outer radiation belt, which means that the energetic electron flux in the outer radiation belt disappeared entirely. Lower-energy electrons (0.3-0.95 $\mathrm{MeV}$ ) remained, although the flux level decreased by two orders of magnitude. The peak portion shifted from $L=4.1$ to $L=3.8$ within several hours.

About 0600 UT on November 4 the energetic electron flux began to increase (see Fig. 2(d)), showing a significant enhancement around $L=3.8$. It should be noted that the $D s t$ value was still decreasing at that time ( 0600 UT); Dst reached its minimum value about 1200 UT on November 4. The electron flux increased continuously, showing the recovery of the outer belt electrons near the peak portion $(L \sim 3.8)$ by the end of November 4 (see Fig. 2(h)). 


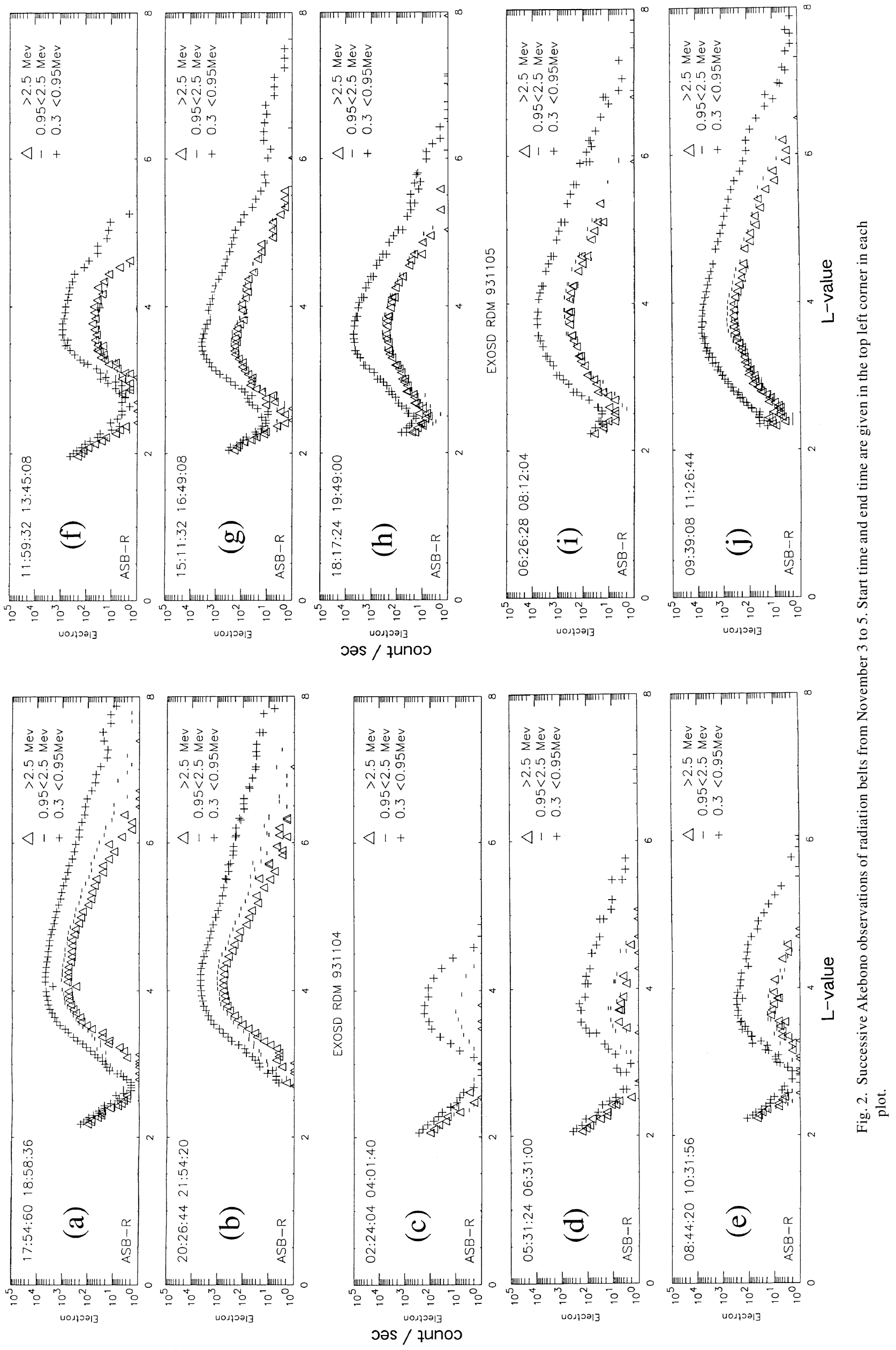



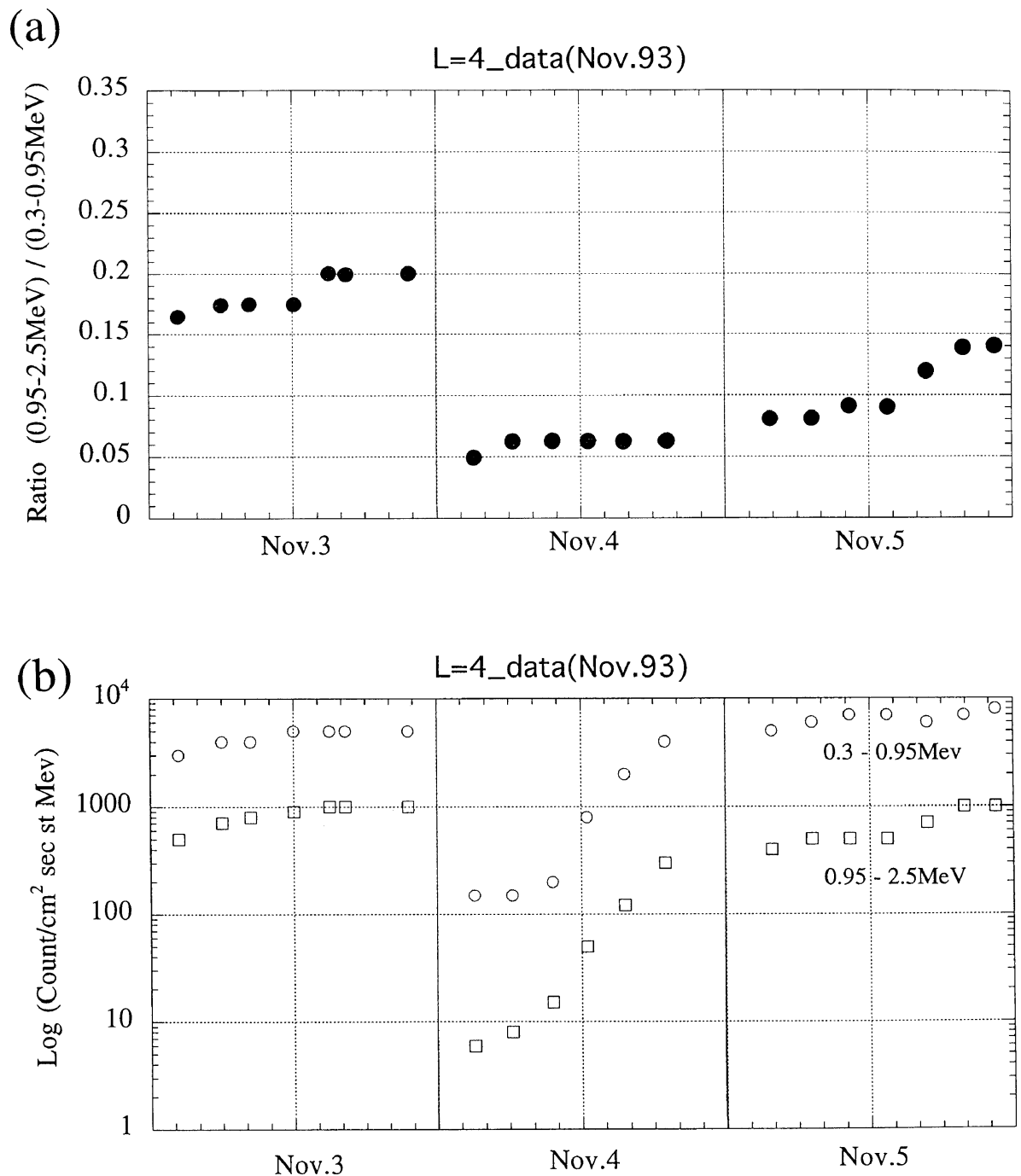

Fig. 3. Ratio (a) and count rates (b) for electrons in two energies; 0.3-0.95 MeV and 0.95-2.5 MeV, at $L=4$ on November 3-5.

Significant enhancement of the energetic electrons near $L=6$ was seen on November 5. By about 1100 UT on November 5 the energetic electron flux recovered near $L \sim 6$ (see Fig. 2(j)) and it was still increasing throughout November 5 and reached its maximum on early November 6.

One more evident thing is the significant shift of the slot region. By 0600 UT on November 5 the minimum-density portion of the slot region shifted from $L=2.8$ to $L=2.5$ because of inward diffusion of the energetic electrons in the outer radiation belt (Mitsui et al., 1999).

The Akebono RDM data give us an impression that the return flux is first seen near the new peak portion of the outer radiation belt and in the lower-energy. To show this more clearly we have plotted, for November 3-5, a flux ratio for electrons in two energies: $0.3-0.95 \mathrm{MeV}$ and 0.95-2.5 MeV. Result is given in Fig. 3(a), where abscissa demonstrates the flux ratio at $L=4$ and ordinate shows the universal time. Figure 3(b) shows the flux level for these two populations. In Fig. 3(b) a sharp decrease in electron flux is evident at the storm onset. The ratio decreased suddenly at the storm onset, and then increased gradually as shown in Fig. 3(a). By late November 5 the ratio was close to its pre-storm value $(\sim 0.15)$. It was also found that the level of the lower energy electrons recovered faster than that of the higher ones.

The corresponding ratio and flux value at $L=5$ are shown in Fig. 4. It should be noted that the flux of higher energy electrons did not recover until about 1400 UT on November 5 (shown in Fig. 4(b)), which means that the recovery of the energetic electrons at $L=5$ was slower than it was at $L=4$. The fluxes increased gradually over two days. It seems to take a longer time for higher-energy electrons in the outer shells to recover.

\section{NOAA Observations}

On the NOAA satellite a particle detector has been installed, observing precipitating electrons in three energy channels: $E_{1}(>30 \mathrm{keV}), E_{2}(>100 \mathrm{keV})$ and $E_{3}(>300 \mathrm{keV})$ (Raben et al., 1995). In early November the NOAA 12 satellite made observations in the morning sector (MLT $~ 8$ h) and the satellite data covering November 1-9 are shown in Fig. 5. Top panel demonstrates a time variation of the electron flux in the energy from $30 \mathrm{keV}$ to $1.1 \mathrm{MeV}$. Second panel shows the electron flux in the energy from $100 \mathrm{keV}$ to $1.1 \mathrm{MeV}$. The third panel is for the flux in the 

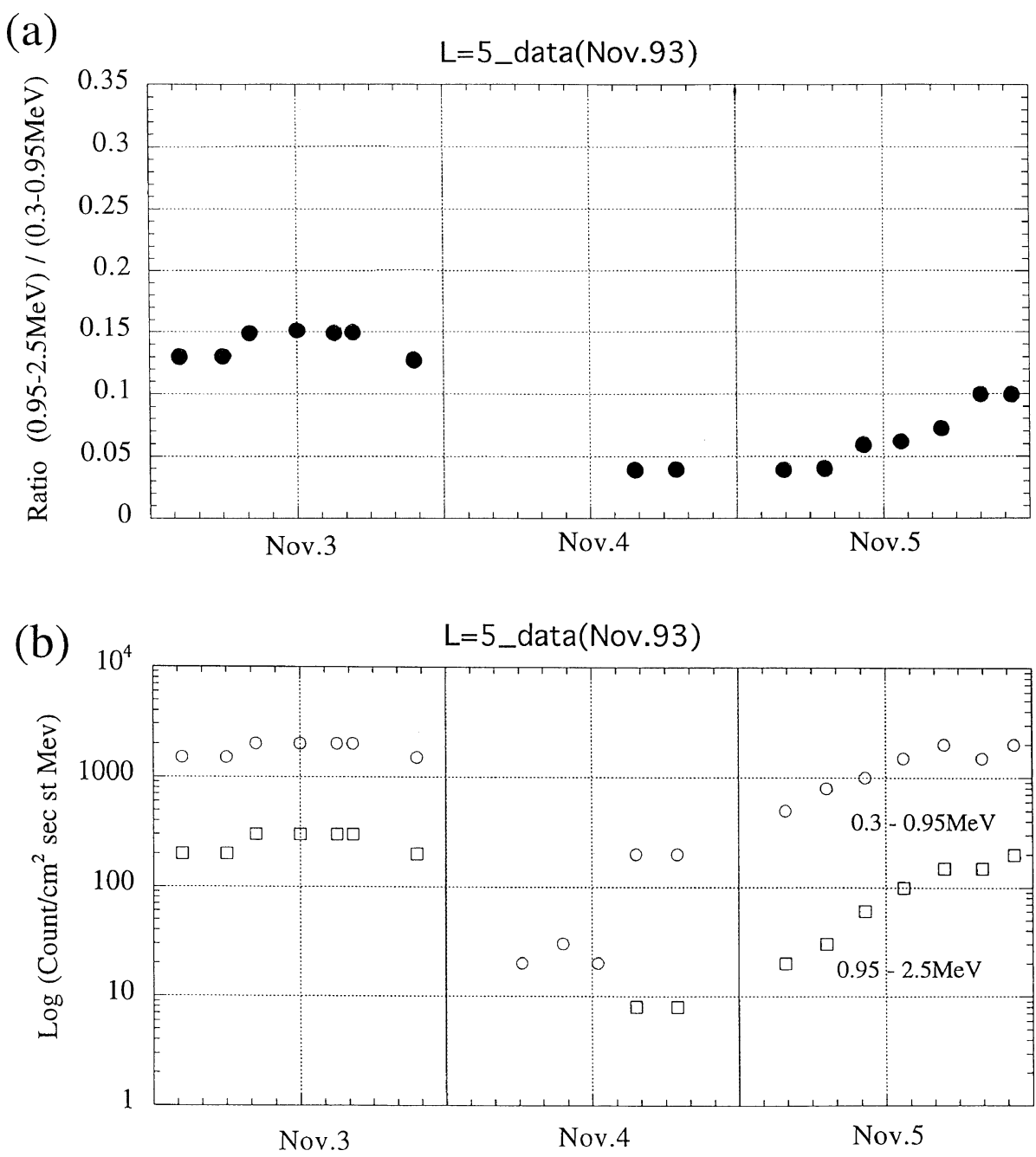

Fig. 4. Ratio (a) and count rates (b) at $L=5$ on November 3-5.

energy range from $300 \mathrm{keV}$ to $1.1 \mathrm{MeV}$. The data shown in Fig. 5(a) were obtained at $L=4$ in the morning sector (MLT $=8 \mathrm{~h}$ ). The calculation of $L$-value in the work in this paper was made by using IGRF.

At the beginning of the magnetic storm the electron flux increased very much. The magnitude of the flux changed approximately from $10^{3}$ (count/sec) to more than $10^{4}$ (count/sec). The increase is larger for lower-energy ( $>30$ $\mathrm{keV}$ and $>100 \mathrm{keV}$ ) electrons and the increase of the higher-energy ( $>300 \mathrm{keV}$ ) electrons is comparatively slow. The increase of the electrons in the energy less than 300 $\mathrm{keV}$ actually served the ring current build up because the timing of the rapid enhancement exactly corresponded to the start of the Dst decrease (see vertical line in the figure). We, therefore, would like to insist that these electrons are ring current electrons.

It is interesting to see that there was a significant time delay for the more energetic ( $>300 \mathrm{keV}$ ) electrons (shown in the third panel in Fig. 5(a)). This delay is more evident when we see the result at $L=4.5$ in the same local time (shown in the third panel in Fig. 5(b)). The number of energetic electrons $(>300 \mathrm{keV})$ showed a gradual increase throughout November 4 and early November 5 .

These data suggest that some of the ring current electrons, which initially have rather low energies $(\sim 100 \mathrm{keV})$, will become more energetic resulting in a gradual increase in the number of energetic $(>300 \mathrm{keV})$ electrons with a significant time delay; half to one day. We are also speculating that there is a way to produce $\mathrm{MeV}$ electron by accelerating $\sim 100 \mathrm{keV}$ ring current electrons, there.

\section{Discussions and Summary}

Considering the Akebono and NOAA data together, we have a scenario to explain the origin of the observed $\mathrm{MeV}$ electrons. The ring current electrons which appeared first and near $L=4$ during the storm main phase serve as "seed" electrons for the subsequent increase of $\mathrm{MeV}$ electron flux there.

Freeman et al. (1998) investigated spectrum characteristics of $\mathrm{MeV}$ electrons and intermediate energy electrons down to about $100 \mathrm{keV}$ at geosynchronous location. They found that electrons from about $100 \mathrm{keV}$ to $1.5 \mathrm{MeV}$ can be characterized by a power law spectrum, suggesting a common source (Freeman et al., 1998). The essential point of our interpretation is that the source electrons are ring current electrons, being consistent with Freeman et al. (1998) with regard to a "common" source. What are the heating/acceleration processes making 

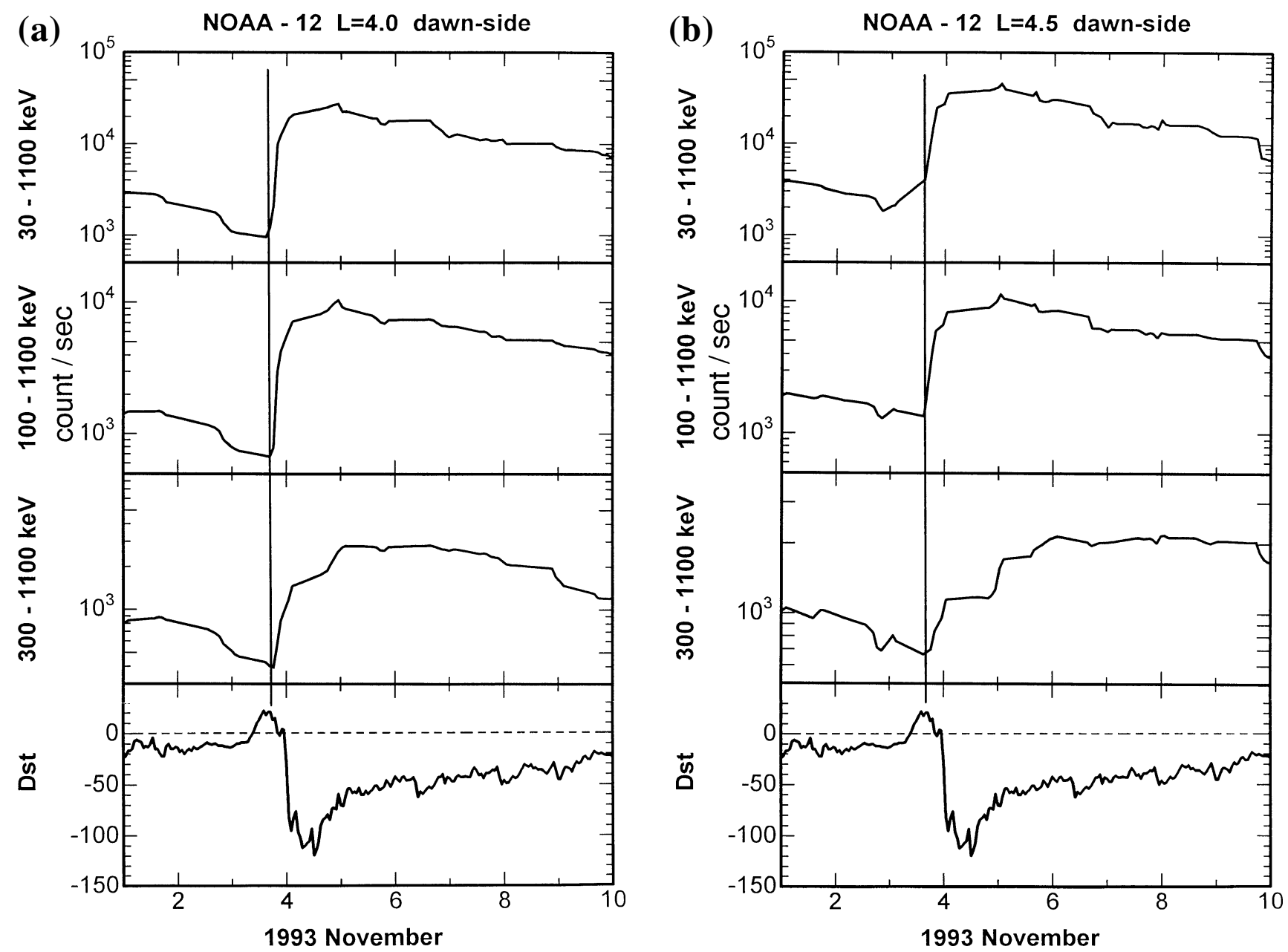

Fig. 5. Count rates for electrons in three energy ranges at $L=4$ (a) and $L=4.5$ (b). Dst index is also shown at the bottom

electrons more energetic and producing relativistic electrons? Possible ones are following; ring current $(D s t)$ effect and a wave-particle interaction. The development of the ring current will reduce magnetic field intensity during the main phase and the delay of the ring current will strengthen the magnetic field. Nakamura et al.(1998) estimated the flux variation of the energetic electrons in the outer belt and concluded that $D s t$ effect cannot fully account for the flux enhancement, inferring some possible acceleration processes are taking place there. We found that the increase in the flux already started during the storm main phase (about 0600 UT on November 4). Even though the Dst was still decreasing at that time, we could see a recovery of the flux of $\mathrm{MeV}$ electrons near $L=4$. This result seems against the Dst mechanism.

We think that local heating processes by wave-particle interacion make these electrons more energetic. The wave activity in a frequency range from $10 \mathrm{kHz}$ to $200 \mathrm{kHz}$ observed by PWS (plasma waves instrument) actually increased in regions ranging from 2 to 6 with regards to $L$ value throughout the storm period. This intensification is due to a sudden reduction of thermal plasma density being waved off by the enhanced convection electric field and a sudden increase of hot particles being transported by the enhanced convection in the storm main phase. We are speculating plasma waves will do significant contribution to the enhancement of the relativistic electrons in the outer radiation belt.

In summary, at the beginning of the storm main phase the rapid increases in the number of electrons with energies from $30 \mathrm{keV}$ to $100 \mathrm{keV}$ was observed near $L=4$ by NOAA spacecraft. At the end of the storm main phase, Akebono observed a significant increase in the flux of the electrons with energies greater than $300 \mathrm{keV}$. Late of the storm main phase and in the storm recovery phase Akebono observed a continuous increase in the flux of $\mathrm{MeV}$ electrons. The increase started near $L \sim 4$ and it propagated to higher- $L$ shelles with a significant time delay. We have suggested that the ring current electrons appearing first around $L=4$ seeded the subsequent increase in the flux of the $\mathrm{MeV}$ electron flux throughout the outer radiation belt. For this to happen, some acceleration processes should make the electrons more energetic.

Acknowledgments. We are grateful to Prof. John Freeman at Rice University for his valuable suggestions. Works in the present paper are made by using Akebono Science Data Base (SDB) released from the Institute of Space and Astronout. Sci., sponsoring agency of the Akebono project. NOAA particle data were supplied by the data center at the National Institute of Polar Research. 


\section{Reference}

Baker, D. N., J. H. Allen, S. G. Kenekal, and G. D. Reeves, Disturbed space environment may have been related to pager satellite failure, EOS, 79, 477-483, 1998.

Freeman, J. W., T. P. O'Brien, A. A. Chan, and R. A. Wolf, Energetic electrons at geostationary orbit during the November 3-4, 1993 storm: Spatial/temporal morphology, characterization by a power law spectrum and representation by an artificial network, J. Geophys. Res., 103, 26251-26260, 1998.

Knipp, D. J.et al., An overview of the early November 1993 geomagnetic storm, J. Geophys. Res., 103, 26197-26220, 1998.

Kozyra, J. U., V. K. Jordanova, J. E. Borovsky, M. F. Thomsen, D. J. Knipp, D. S. Evans, D. J. McComas, and T. E. Cayton, Effects of a high-density sheet on ring current development during the November 2-6, 1993, magnetic storm, J. Geophys. Res., 103, 26285-26305, 1998.

Li, X., D. N. Baker, M. Temerin, T. E. Cayton, G. D. Reeves, R. A. Christensen, J. B. Blake, M. D. Looper, R. Nakamura, and S. G Kenekal, Observations of the outer zone electron variation during the November 3-4, 1993, magnetic storm, J. Geophys. Res., 102, 1412314140, 1997.

Mitsui, C., A. Tsutai, T. Nagai, T. Kohno, and F. Makino, Energetic electron filling of the slot region: EXOS-D observations, Earth
Planets Space, 1999 (submitted).

McIlwain, C. E., Ring current effects on trapped particles, J. Geophys. Res., 71, 3623-3628, 1966.

Nakamura, R., K. Kamei, and Y. Kamide, SAMPEX observation of storm-associated electron flux variation in the outer radiation belt, $J$. Geophys. Res., 103, 26261-26269, 1998.

Obara, T., M. Den, T. Nagatsuma, and E. Sagawa, Enhancement of the trapped radiation electrons at $6.6 \mathrm{Re}$ during the storm recovery phase-results from GMS/SEM observation-, Proceeding on NIPR Symposium on Upper Atmospheric Phys., 12, 86-93, 1998.

Raben, V. J., D. E. Evans, H. H. Sauer, S. R. Sahm, and M. Huynh, TIROS/NOAA satellite space environment monitor data archive documentation: 1995 update, NOAA Tech. Memo. ERL SEL-86, 1995.

Yokoyama, N. and Y. Kamide, Statistical nature of geomagnetic storms, J. Geophys. Res., 102, 14215-14222, 1997

T. Obara (e-mail: T.Obara@crl.go.jp), T. Nagatsuma (e-mail: tnagatsu@crl.go.jp), M. Den (e-mail: den@crl.go.jp), Y. Miyoshi (e-mail: miyoshi@pparc.geophys.tohoku.ac.jp), and A. Morioka (e-mail: morioka@pparc.geophys.tohoku.ac.jp) 